\title{
Prediction of Growth of Turbulence in a Uniformly Sheared Flow Using the Vortex-in-Cell Method
}

\author{
R. Abdolhosseini and R.E. Milane \\ Department of Mechanical Engineering \\ University of Ottawa, \\ Ottawa, Canada
}

\begin{abstract}
A mixed Lagrangian-Eulerian description of the evolution of the discretized vorticity field is used for the numerical prediction of incompressible, uniformly sheared turbulent flows. In this new application a combination of several adjacent mixing layers simulates the initial condition used to generate the uniformly sheared flow as done in some of the experimental methods in wind tunnels. The two dimensional vortex dynamics method which has been extensively used for mixing layer analysis is adopted due to the similarities in the structure and the method of generating mixing layers and uniformly sheared flows. The governing equations are the vorticity and the continuity equations. The vorticity field is represented by a set of vortex elements which moves by the induced velocity field. To treat the large number of vortex elements at a reasonable computer cost, the velocity field is calculated by the vortex-in-cell (VIC) method, in which 48000 vortices move through a fixed Eulerian mesh system with $160 \times 16$ grid points. Statistical results were obtained by time averaging the unsteady solution. The flow field was calculated for the shear rate $d U / d y=67 \mathrm{~s}^{-1}$ and centerline velocity $U_{c}=425 \mathrm{~cm} / \mathrm{s}$.
\end{abstract}

\section{Introduction}

Unlike the numerical techniques that apply an Eulerian system to solve the equations of turbulent flow, the vortex methods describe the flow in a Lagrangian fashion. Vortex methods have proven their validity in the numerical simulation of mixing layers and have been used extensively to simulate incompressible flows, see e.g., Ghoniem and $\mathrm{Ng}$ [8] and Inoue [10]. The objective of this study is to extend the prediction of mixing layers using the two dimensional vortex method to the uniformly sheared flows.

Previous experimental and numerical investigations in the uniformly sheared turbulent flows and spatially growing mixing layers have indicated similarities in the structure of their vorticity field. Bernal and Roshko [2] showed experimentally that unforced mixing layers exhibit hairpin vorticity structures, and Rogers and Moin [15] reported hairpin structures in the numerical prediction of uniformly sheared flows. There are also similarities in the method of generating uniformly sheared flows and mixing layers experimentally in wind tunnels. Figure 1 shows a schematic configuration of one of the widely used methods of producing uniformly sheared flows in wind tunnels. A set of parallel splitter plates producing channels of flow with various resistances, produced by different screens, initialize the flow field. It is therefore expected that the prediction of the uniformly sheared flow using the two-dimensional vortex method is comparable to the mixing layer.

In spite of the advantages of the pure Lagrangian vortex methods in avoiding the diffusion error, their computational cost could be prohibitive. The computational cost is more restrictive for the 
uniformly sheared flows because the number of vortices is much higher than mixing layers for two reasons. Firstly, several shear layer each corresponding to a splitter plate are required, whereas one is sufficient for the mixing layer. Secondly, the computational domain must be longer to obtain a fully developed flow due to lower shear rate and velocity difference between the layers.

In order to reduce the computational cost, the vortex-in-cell (VIC) method (Christiansen [6]) is used. This method is a hybrid of the Lagrangian and Eulerian methods. In the VIC method vortex markers are traced using a Lagrangian system but these markers move through an Eulerian mesh system.

\section{Background}

There have been numerous experiments attempting to generate and to investigate various aspects of uniformly sheared turbulent flows. The first attempt using wind tunnel was made by Rose [17]. His results showed that the integral length scales and Taylor micro scales vary in the cross-stream direction due to the mean shear generator mechanism. This difficulty was overcome by Champagne et al. [3] who used a setup consisting of a row of equally spaced channels with adjustable internal resistance. Harris et al. [9] were the first to recognize that the value of the non-dimensional time $(\tau)$ determines whether or not the Reynolds stresses and turbulent kinetic energy are growing with the streamwise direction. The non-dimensional time $\tau$ is $K_{s} x$, where $K_{s}$ is the flow generator constant defined as $1 / U_{c}(d U / d y), U_{c}$ is the centerline mean velocity, $d U / d y$ is the shear rate and $x$ is the streamwise coordinate. Tavoularis [21] showed that the turbulent kinetic energy grows exponentially in the asymptotic development range as $e^{\kappa \tau}$ where $\kappa$ was found to be about 0.1 for the high shear rate experiments by Tavoularis and Karnik [23]. Rohr et al. [16] produced a uniformly sheared flow using a ten-layer closed loop water tunnel. The same Reynolds number with a much smaller mean velocity was obtained because the kinematic viscosity of water is about fifteen times smaller than air. The authors pointed out that the differences in the growth rates between the experimental results is reduced if the non-dimensional time $(\tau)$ is used instead of the streamwise coordinate $(x)$.

Rose [17], Champagne et al. [3] and Mulhearn and Luxton [12] obtained non-growing downstream Reynolds stresses because of the small values of $\tau(\tau<5)$, whereas Harris et al. [9], Tavoularis and Corrsin [22], Rohr et al. [16] and Tavoularis and Karnik [23] showed that giving sufficient non-dimensional time $(\tau>8)$, the Reynolds stresses increase monotonically. In a more recent investigation, Souza [20] conducted measurements in a high speed uniformly sheared turbulent flow with $d U / d y$ ranging from 436 to $705 \mathrm{~s}^{-1}$ and Mach number below 0.2 . Her measurements showed qualitatively the same results for downstream growth rate of the turbulent kinetic energy. However the Reynolds shear stress was lower than the experiments with the lower shear rate, e.g., $84 \mathrm{~s}^{-1}$ in Tavoularis and Karnik [23].

Direct numerical simulations have been used to predict the uniformly sheared flows. Shirani et al. [19] used the spectral method and concentrated on the case where the mean scalar gradient was imposed in the same direction as the mean shear. Since their computation used a relatively coarse grid, no effort was made to search for organized turbulent structure. Feiereisen et al. [7] applied the spectral method to simulate incompressible and compressible sheared flows. They did not report the streamwise variation of the Reynolds stresses, but they compared the pressure-strain terms with the predictions of turbulent models. Rogers and Moin [15] simulated the structure of vorticity field in uniformly sheared turbulent flows. They used the DNS of three dimensional, incompressible, time dependent Navier-Stokes equations using Rogallo's [13] computer code. The initial velocity field was random, subject to the constraint of continuity and a specified isotropic energy spectrum. 
They found in all cases, the presence of coherent vortical structure. The results for three different flow fields showed that the vorticity tends to be aligned with the direction of positive strain. The streamwise variation of the component energy ratios are in agreement with the experimental results of Tavoularis and Corrsin [22] in the range $8<\tau<12$. Lee et al. [11] investigated the structure of highly sheared homogeneous turbulent flows using the pseudo-spectral code developed by Rogallo [13]. They applied a combination of DNS and RDT to calculate the instantaneous flow fields. The initial velocity field was isotropic as in Rogers and Moin [15]. Their results showed that turbulent kinetic energy is increasingly concentrated in the streamwise component with increasing downstream position, suggesting that a prolonged shear $(\tau \rightarrow \infty)$ would produce asymptotically a one component turbulence.

To the authors knowledge, vortex methods have not yet been used to simulate uniformly sheared flows and the present work is the first attempt to apply a mixed Lagrangian-Eulerian approach to this problem.

\section{Governing equations}

\subsection{Vorticity transport equation}

The vorticity transport equation is written as (see e.g., Tennekes and Lumley [24]):

$$
\frac{\partial \omega_{i}}{\partial t}+u_{j} \frac{\partial \omega_{i}}{\partial x_{j}}=\omega_{j} \frac{\partial u_{i}}{\partial x_{j}}+\nu \frac{\partial^{2} \omega_{j}}{\partial x_{j} \partial x_{j}}
$$

where $u$ is the velocity vector, $\omega$ is the vorticity vector and $\nu$ is the kinematic viscosity. The left hand side corresponds to the time rate of change of vorticity and the convective terms. The first term on the right hand side corresponds to the vortex stretching and is nil in two dimensional cases since the vorticity vector $\omega$ is perpendicular to the gradient of $u$. In this case, the vorticity transport equation is written as

$$
\frac{\partial \omega_{i}}{\partial t}+u_{j} \frac{\partial \omega_{i}}{\partial x_{j}}=\nu \frac{\partial^{2} \omega_{j}}{\partial x_{j} \partial x_{j}}
$$

Since $\mathbf{u}=\mathbf{u}(x, y, t)$, the vorticity vector reduces to one component in the $z$ direction perpendicular to the $x y$ plane (the plane of motion) as

$$
\omega=\frac{\partial v}{\partial x}-\frac{\partial u}{\partial y}
$$

Using the continuity equation $\nabla \cdot \mathbf{u}=0$, the velocity $\mathbf{u}$ can be expressed in terms of a streamfunction $\psi$ such that $u=\partial \psi / \partial y, v=-\partial \psi / \partial x$. Combining these expressions with Eq. 3, a Poisson equation is obtained

$$
\nabla^{2} \psi=-\omega_{z}
$$

Equation 4 is used to determine the velocity field from the vorticity distribution given by the right hand side.

\subsection{Boundary and initial conditions}

The computational domain consists of a rectangular region covered by a uniform mesh system (Fig. 2). Several adjacent splitter plates generating mixing layers are used to simulate the initial condition 
of the uniformly sheared flow. Co-flowing streams with uniform but different stream velocities are discharged from the splitter plates. This method is similar to the experimental production of uniformly sheared flows. Slip wall boundaries are assumed for the top and bottom boundaries. The inflow (left) boundary condition is of Neuman type. At this boundary a combination of stepwise uniform velocity between splitter plates gives a linearly distributed streamfunction with slopes equal to local velocity as

$$
\left.\frac{\partial \psi}{\partial y}\right|_{1, j}=U_{1, j}
$$

The outflow boundary condition is similar to the inflow boundary condition. A boundary condition of Dirichlet type is used for the top and bottom boundaries. For the bottom boundary where $y=1$ the value of $\psi$ is

$$
\psi_{1, j}=U_{\text {bottom }}
$$

and for the top boundary the value of $\psi$ is

$$
\psi_{i, N}=U_{\text {bottom }} y_{\text {bottom }}+\Sigma U_{k} S+U_{\text {upper }}\left(y_{1, N}-y_{\text {upper }}\right)
$$

where $S$ is the distance between splitter plates, $U_{\text {bottom }}$ and $U_{\text {upper }}$ are the velocities below the bottom splitter plate and above the upper splitter plate, respectively, $y_{\text {bottom }}$ and $y_{\text {upper }}$ are the coordinates of the bottom splitter plate and the upper splitter plate, respectively, $U_{k}$ is the velocity between splitter plate $k$ and $k+1$. The velocity discontinuity across the splitter plates is simulated using shear layers. Each shear layer is discretized into a row of equidistant vortices. The velocity difference $\Delta U$ between the streams defines the circulation of the vortices in such a way that the Kutta condition is satisfied. The circulation of each vortex is $\Gamma=\Delta U \cdot d$, where $d$ is the initial distance between neighbouring vortices. At the edge of each splitter plates a vortex is introduced at different time interval $d / U_{i}$ where $U_{i}$ is the arithmetic average of the velocities above and below the splitter plate $i$. The vortices move freely in and out of the domain and their effect is cancelled if they are outside the domain. Therefore the number of vortices is not constant in the computational domain.

\section{Numerical methods and solution procedure}

The following two fractional step are used to solve the vorticity transport equation (Eq. 2):

$$
\begin{aligned}
& \frac{\partial \omega_{z}}{\partial t}+u \frac{\partial \omega_{z}}{\partial x}+\nu \frac{\partial \omega_{z}}{\partial y}=0 \\
& \frac{\partial \omega_{z}}{\partial t}=\nu\left(\frac{\partial^{2} \omega_{z}}{\partial x^{2}}+\frac{\partial^{2} \omega_{z}}{\partial y^{2}}\right)
\end{aligned}
$$

In the first fractional step (Eq. 8), the convection due to the action of the vortices is obtained from the solution of the Poisson equation (Eq. 4) using the VIC method. In the second fractional step (Eq. 9), the diffusion of vorticity is solved using the random walk method (Chorin [5]). 


\subsection{Vortex-in-cell (VIC) method}

The vorticity allocated $\left(\omega_{i}\right)$ to each of the four nodal points around each vortex (Fig. 3$)$ is calculated using an area-weighting scheme (Baker [1]) as:

$$
\omega_{i}=\frac{\Gamma A_{i}}{\Delta x \Delta y} \quad i=1,2,3,4
$$

where the $A_{i}$ 's are the areas shown in Fig. $3, \Delta x$ and $\Delta y$ are the mesh sizes, and $x_{n}$ and $y_{n}$ are the coordinates of a vortex. The Poisson equation (Eq. 4) is solved to obtain the streamfunction $\psi_{i, j}$ at the nodes by a finite difference approximation. The velocity at the nodal points is then calculated from $u=\partial \psi / \partial y, v=-\partial \psi / \partial x$ using a finite difference approximation. The velocity of each vortex in the mesh is calculated using a bilinear interpolation as

$$
\begin{aligned}
& u_{n}=\left(\Sigma u_{i} A_{i}\right) / \Delta x \Delta y \\
& v_{n}=\left(\Sigma v_{i} A_{i}\right) / \Delta x \Delta y
\end{aligned}
$$

where $u_{i}$ and $v_{i}$ are the components of the velocity calculated at the nodal points.

\subsection{Displacement of the vortices}

The trajectory $\chi$ of each vortex is calculated by integrating

$$
\frac{d \chi}{d t}=\mathbf{u}(\chi(x, y), t)
$$

using the first order Euler scheme in time step $\Delta t$ as:

$$
\begin{aligned}
& x_{n}(t+\Delta t)=x_{n}(t)+u_{n} \Delta t+\eta_{1} \\
& y_{n}(t+\Delta t)=y_{n}(t)+v_{n} \Delta t+\eta_{2}
\end{aligned}
$$

where $\eta_{1}$ and $\eta_{2}$ are random variables with zero mean and standard deviation $\sqrt{2 \nu \Delta t}$ and $\nu$ is the kinematic viscosity.

\subsection{Solution procedure}

The solution procedure consists of the following steps:

1. Initialization by assuming distributions of $\psi$ and $\omega_{z}$ together with the boundary conditions.

2. Solving the Poisson equation for $\psi$ by sweeping all nodes from left to right and line by line, and using the SOR method with a second order central difference scheme.

3. Computing the velocities $u$ and $v$ at the nodal points using second order central difference schemes.

4. Calculating the velocities of the vortices using the bilinear interpolation.

5. Advancing the vortices in time using Eqs. 13a,b.

6. Applying the area weighting scheme to redistribute the vorticities at the nodal points (Eq. $10)$.

7. Introducing a vortex at the splitter plates and deleting the effect of vortices that pass the downstream boundary.

The unsteady solution is obtained by repeating the calculations from step 2 to step 7 . 


\section{Parameters}

Ten splitter plates are used to initialize the flow at the inflow boundary. The flow field is calculated for the shear rate $d U / d y=67 \mathrm{~s}^{-1}$ and the centerline velocity $U_{c}=425 \mathrm{~cm} / \mathrm{s}$, and the flow generator constant $K_{s}=0.157 \mathrm{~cm}^{-1}$. The kinematic viscosity, $\nu=0.2 \times 10^{-2} \mathrm{~cm}^{2} / \mathrm{s}$ corresponds to a slightly viscous flow. This ensures that the random walk method is properly used as discussed by Chang [4]. A uniform mesh system with $160 \times 16$ grid points in which the grid size is $\Delta x=\Delta y=1 \mathrm{~cm}$ is used. Each shear layer is initially discretized into 4800 vortices separated by a distance $d=0.033 \mathrm{~cm}$. Thus a total of 48000 vortices are tracked in each time step. The circulation of each vortex is $\Gamma=2.5 \times 10^{-2} \mathrm{~m}^{2} / \mathrm{s}$. A time step of $4.371 \times 10^{-5} \mathrm{~s}$, calculated as $\Delta t=d / U_{10}$ is used, where $d$ is the initial spacing between the vortices and $U_{10}$ is the arithmetic average of the velocities below and above the tenth splitter plate where the velocity is the highest. Initially the vortex sheets are perturbed by moving the first vortex on each sheet a short distance, $0.3 d$ in the lateral direction. The total number of time steps used is 45000 , with the last 30000 time steps used to calculate the mean velocity and the Reynolds stresses.

\section{Results and discussion}

Flow field: The streaklines of the vortices is shown in Fig. 4 from $x=0 \mathrm{~cm}$ (edge of the splitter plates) to $160 \mathrm{~cm}$. The roll-up of the vortex sheet, vortex pairing and amalgamation are responsible for the initial flow development. The lower layers develop earlier than the upper layers due to their lower velocities. The stretching of the vorticity gradient, which can be inferred from the vorticity contours in Fig. 5, generates small scales.

Mean velocity profile: The profiles of the normalized mean velocity in the developed region at several downstream stations are shown in Fig. 6. The normalized mean velocity is plotted versus $\left(y-y_{0}\right) / H$, where $y_{0}$ is the ordinate of the fifth splitter plate from the bottom splitter plate and $H$ is half of the width of the computational domain; $\left(y-y_{0}\right) / H=-0.5$ corresponds to the first splitter plate (bottom), $\left(y-y_{0}\right) / H=0.625$ corresponds to tenth splitter plate and $\left(y-y_{0}\right) / H=0.0625$ corresponds to the centerline. The mean velocity profiles are homogeneous in the streamwise positions and vary linearly with the cross-stream positions.

Reynolds stresses: The rms velocity fluctuations and the Reynolds shear stress profiles, normalized by the centerline velocity at several downstream stations are shown in Figs. 7 to 9 . Figures 7 and 8 show that the rms $u^{\prime}$ and the rms $v^{\prime}$ are nearly homogeneous in the lower layers in the range $-0.5 \leq\left(y-y_{0}\right) / H \leq-0.1$. The non-homogeneity in the range $-0.1 \leq\left(y-y_{0}\right) / H \leq-0.625$ is due to the late development of the upper layers as shown by the streaklines in Fig. 4. The homogeneity of the Reynolds shear stress in Fig. 9 is better than the rms $u^{\prime}$ and rms $v^{\prime}$. The growth of the Reynolds stresses with the downstream position is evident and the trend will be discussed later in this section.

Figures 10 to 12 show the downstream growth of the Reynolds stresses at centerline. Also shown are the experimental results of Tavoularis and Karnik [23] with $K_{s}=0.064 \mathrm{~cm}^{-1}$ and air, Tavoularis and Corrsin [22] with $K_{s}=0.035 \mathrm{~cm}^{-1}$ and air, and Rohr [16] with $K_{s}=0.047,0.48$ and $0.61 \mathrm{~cm}^{-1}$ and water at the centerline where the Reynolds stresses are almost maximum (the farthest region from the walls). The experimental results show that the growth rate of Reynolds stresses is insensitive to the value of $K_{s}$ when plotted versus $\tau$. Note that $\tau$ is a non-dimensional parameter showing the steamwise distance as discussed in Section 2. The value of $K_{s}=0.157 \mathrm{~cm}^{-1}$ used in 
Table I: Slopes of the streamwise growth rate of Reynolds stresses and turbulent kinetic energy.

\begin{tabular}{lcccccc}
\hline \hline & $\begin{array}{c}U_{c} \\
(\mathrm{~cm} / \mathrm{s})\end{array}$ & $\begin{array}{c}d U / d y \\
(1 / \mathrm{s})\end{array}$ & $\begin{array}{c}K_{s} \\
(1 / \mathrm{cm})\end{array}$ & $\begin{array}{c}\text { Slope rms } \\
u^{\prime} / U_{c}\end{array}$ & $\begin{array}{c}\text { Slope rms } \\
v^{\prime} / U_{c}\end{array}$ & $\begin{array}{c}\text { Slope } \\
\left(q / U_{c}\right)^{2}\end{array}$ \\
\hline Exp. Tavoularis and Karnik [23] & 1300 & 84 & 0.064 & 0.05 & 0.041 & 0.094 \\
Exp. Tavoularis and Corrsin [22] & 1240 & 46.8 & 0.037 & 0.07 & 0.061 & - \\
Exp. Rohr et al. [16] & 20.1 & 1.23 & 0.061 & & & \\
& 20.2 & 0.96 & 0.047 & 0.062 & 0.068 & - \\
& 26.4 & 1.29 & 0.048 & & & \\
Present simulation & 425 & 67 & 0.157 & 0.045 & 0.043 & 0.086 \\
\hline \hline
\end{tabular}

the simulation is about three to five times the reported experimental values. This is done to reduce the computational cost by reducing the computational domain in the streamwise direction $(x)$. However the values of the non-dimensional time $\tau$ which plays a fundamental role in characterizing the uniformly sheared flow (Rohr et al. [16]), is in the range of the experimental data reported.

The rms longitudinal velocity fluctuations $\left(u^{\prime} / U_{c}\right)$ in Fig. 10 exhibits two regions for the growth rate. The undeveloped region (closer to the splitter plates) where the growth rate is faster than in experiments and a developed region $(\tau \geq 15)$ where the growth rate reaches an asymptotic state which is in good agreement with the experiment. Table I shows that the simulated slope is close to the experiments in particular to the experiment of Tavoularis and Karnik [23]. The offset between the numerical and the experimental results in the developed region is due to the difference in the inflow conditions. In the numerical simulations the inflow boundary condition has no turbulence, while in the experimental cases the shear flow is generated by a set of splitter plates with grids of various resistance which are inherently turbulence generator. The grid generated turbulence dominates the inlet region and decays after a while, depending on the type of generators. As explained in good details by Rohr et al. [16], the grid generated turbulence has a continued effect on the downstream growth of turbulence, depending on the grid size, the distance between the grids and the inlet flow condition. The grid generated turbulence introduces an offset in the Reynolds stresses profiles. In the asymptotic region (developed region), the magnitude of the Reynolds stresses are directly related to the initial conditions but their growth rate is independent of the initial conditions when plotted versus $\tau$. Downstream growth rates of the lateral velocity fluctuations and the Reynolds shear stress are shown in Figs. 11 and 12, respectively. The conclusions are similar to those presented for the growth rate of the longitudinal velocity fluctuations except for the Reynolds shear stress $\left(-u^{\prime} v^{\prime}\right)$ which shows non-monotonic growth at some locations $\tau$.

The downstream growth of the turbulent kinetic energy $q^{2}=\left(\left(\operatorname{rms} u^{\prime}\right)^{2}+\left(\operatorname{rms} v^{\prime}\right)^{2}\right) / 2$ is plotted in Fig. 13. The asymptotic growth rate of the turbulence kinetic energy is in good agreement with the experimental results. The experimental and numerical values are 0.094 and 0.086 respectively (see Table I) which are close to the value $\kappa \approx 0.1$ obtained for the high shear rate experiments (Tavoularis and Karnik [23]). The offset is due to the grid generated turbulence in the experimental cases. Figure 14 show the turbulent kinetic energy normalized with $q_{r}$, where $q_{r}$ is the turbulent kinetic energy near the end of the computational domain which corresponds to the end of the test section of wind tunnel in the experimental cases. The growth of the turbulent kinetic energy in the developed regions is close to the experimental results.

The effect of the offset due to the different initial conditions when comparing experimental results with simulation, can be cancelled by comparing the fractional energy components $K_{11}=$ $\left(\mathrm{rms} u^{\prime} / q\right)^{2}, K_{22}=\left(\operatorname{rms} v^{\prime} / q\right)^{2}$ and $K_{12}=-u^{\prime} v^{\prime} / q^{2}$ in the asymptotic regions $(\tau>15)$. Figure 15 
shows that the numerical value of $K_{11}=0.38$ is $25 \%$ less than the experimental value of $K_{11}=$ $0.51 \pm 0.04$. The numerical value of $K_{22}=0.62$ is $160 \%$ the experimental value of $K_{22}=0.22 \pm 0.02$. This result is comparable to the unforced mixing layer prediction which shows that the rms lateral velocity fluctuations is twice the experimental value, e.g., Ghoniem and $\mathrm{Ng}$ [8]. The numerical value of $K_{12}=0.08$ is $33 \%$ less than the experimental value of $K_{12}=0.12 \pm 0.01$.

\section{Concluding remarks}

The vortex-in-cell (VIC) method is used to predict the flow field, and the growth rates of the Reynolds stresses and the turbulent kinetic energy of the uniformly sheared flow. Two regions of growth rate are predicted. Region closer to the splitter plates where the growth rate is faster than the experiment, followed by the asymptotic region where the growth rate is in agreement with experiment. In the asymptotic region the predicted growth rate of turbulent kinetic energy follows an exponential function as $e^{\kappa \tau}$ with $\kappa=0.086$ which is close to the experimental results.

The absolute values of the Reynolds stresses and turbulent kinetic energy differ from the experiment by an offset due to existing grid generated turbulence in experiments or probably due to the approach used in the simulation. In the asymptotic range, the fractional energy components of longitudinal velocity fluctuations and Reynolds shear stress are in close agreement with experiment whereas the fractional energy component of the lateral velocity fluctuations is $160 \%$ higher than experiment. The differences in the fractional energy components between numerical prediction and experiment are comparable to the prediction of the unforced mixing layer using the two dimensional vortex method.

\section{References}

[1] Baker, G.R., "The Cloud-in-Cell Technique Applied to the Roll-Up of the Vortex Sheets," $J$. Comp. Phys., 31, pp. 76-95, 1979.

[2] Bernal, L.P. and Roshko, A., "Streamwise Vortex Structure in Plane Mixing Layers," J. Fluid Mech., 170, pp. 499-525, 1986.

[3] Champagne, F.H., Harris, V.G. and Corrsin, S., "Experiments in Nearly Homogeneous Turbulent Shear Flows," J. Fluid Mech., 41, pp. 81-140, 1970.

[4] Chang, C.C., "Random Vortex Methods for the Navier-Stokes Equations," J. Comp. Phys., 76, pp. 281-300, 1988.

[5] Chorin, A.J., "Numerical Study of Slightly Viscous Flow," J. Fluid Mech., 57, pp. 785-796, 1973.

[6] Christiansen, J.P., "Numerical Simulation of Hydromechanics by the Method of Point Vortices," J. Comp. Phys., 13, pp. 363-379, 1973.

[7] Feiereisen, W.J., Reynolds, W.C. and Ferziger, J.H., "Numerical Simulation of a Compressible, Homogeneous, Turbulent Shear Flow," Department of Mech. Eng'n Rep. TF-13, Stanford University, Stanford, California, 1981.

[8] Ghoniem, A.F. and Ng, K.K., "Numerical Study of the Dynamics of a Forced Shear Layer," Phys. Fluids, 30 (3), pp. 706-721, 1987. 
[9] Harris, V.G., Graham, A.H. and Corrsin, S., "Further Experiments in Nearly Homogeneous Turbulent Shear Flow," J. Fluid Mech., 81, pp. 657-687, 1977.

[10] Inoue, O., "Double Frequency Forcing on Spatially Growing Mixing Layers," J. Fluid Mech., 234, pp. 553-581, 1992.

[11] Lee, M.J., Kim, J. and Moin, P., "Structure of Turbulence in High Shear Rate," J. Fluid Mech., 216, pp. 561-583, 1990.

[12] Mulhearn, P.J. and Luxton, R.E., "The Development of Turbulent Structure in a Uniform Shear Flow," J. Fluid Mech., 68, pp. 577-590, 1975.

[13] Rogallo, R., "Numerical Experiments in Homogeneous Turbulence," NASA Technical Memo. 81315, 1981.

[14] Rogallo, R. and Moin, P., "Numerical Simulation of Turbulent Flows," Ann. Rev. Fluid Mech., 16, pp. 99-137, 1984.

[15] Rogers, M.M. and Moin, P., "The Structure of Vorticity Field in Homogeneous Turbulent Flows," J. Fluid Mech., 176, pp. 33-66, 1987.

[16] Rohr, J.J., Itsweire, E.C., Helland and Van-Atta, "An Investigation of the Growth of Turbulence in a Uniform Mean Shear Flow," J. Fluid Mech., 187, pp. 1-33, 1988.

[17] Rose, W.G., "Results of an Attempt to Generate a Homogeneous Turbulent Shear Flow," J. Fluid Mech., 25, pp. 97-120, 1966.

[18] Sarpkaya, E., "Vortex Element Methods for Flow Simulation," Adv. Appl. Math., 31, pp. 113247, 1994.

[19] Shirani, E., Ferziger, J.H. and Reynolds, W.C., "Mixing of a Passive Scalar in Isotropic and Sheared Homogeneous Turbulence," Dept. Mech. Eng'n Rep. TF-15, Stanford University, Stanford, California, 1981.

[20] Souza, F., Experiments in Highly Sheared Nearly Homogeneous Turbulence, M.Sc. Thesis, Dept. Mech. Eng'n, Univ. of Ottawa, Ottawa, Canada, 1993.

[21] Tavoularis, S., "Asymptotic Laws for Transversely Homogeneous Turbulent Shear Flows," Phys. Fluids, 28 (3), pp. 999-1001, 1985.

[22] Tavoularis, S. and Corrsin, S., "Experiments in Nearly Homogeneous Turbulent Shear Flow with a Uniform Mean Temperature Gradient," J. Fluid Mech., 104, pp. 311-367, 1981.

[23] Tavoularis, S. and Karnik, U., "Further Experiments on the Evolution of Turbulent Stresses and Scales in Uniformly Sheared Turbulence," J. Fluid Mech., 204, pp. 457-478, 1989.

[24] Tennekes, H. and Lumley, J.L., MIT Press, A First Course in Turbulence, Cambridge, Mass., 1978. 


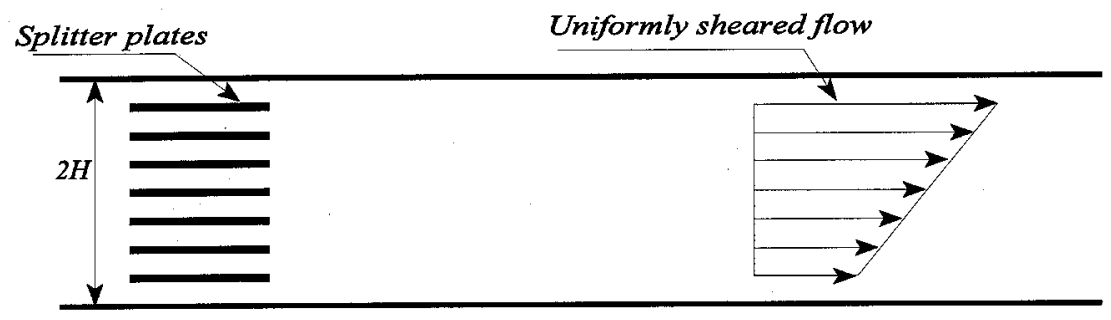

Figure 1: Schematic diagram showing the shear generator and velocity profile.

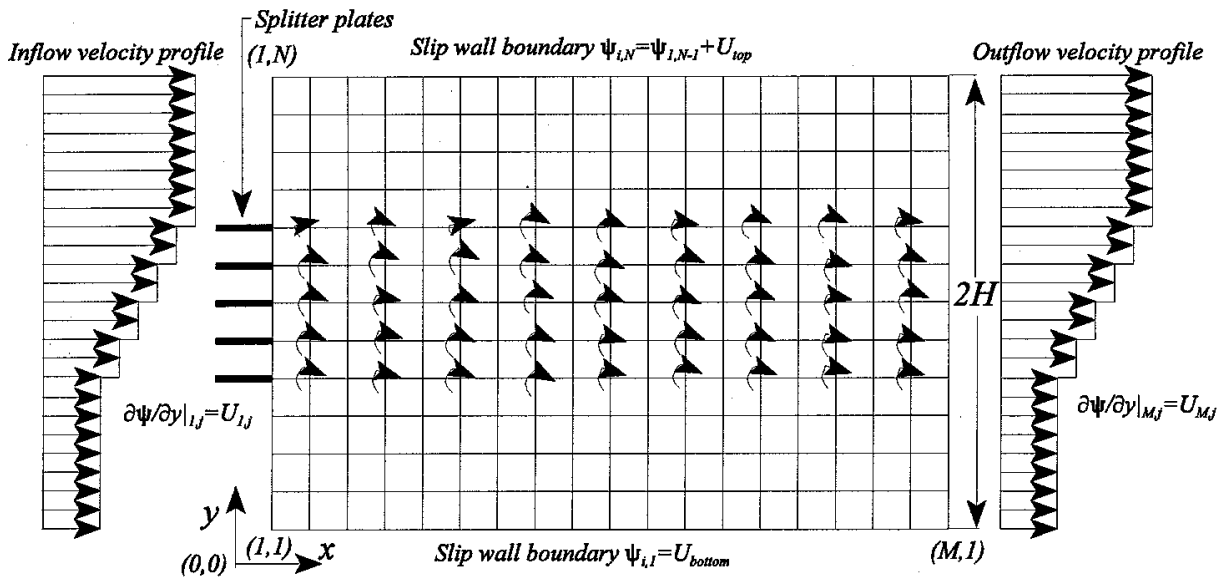

Figure 2: Grid system and boundary conditions.

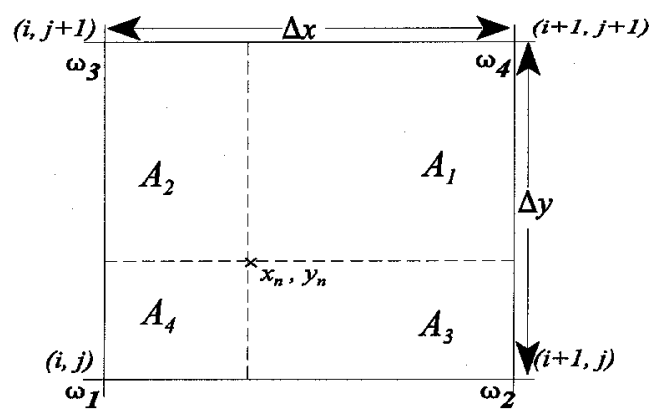

Figure 3: Area weighting scheme for the VIC method.

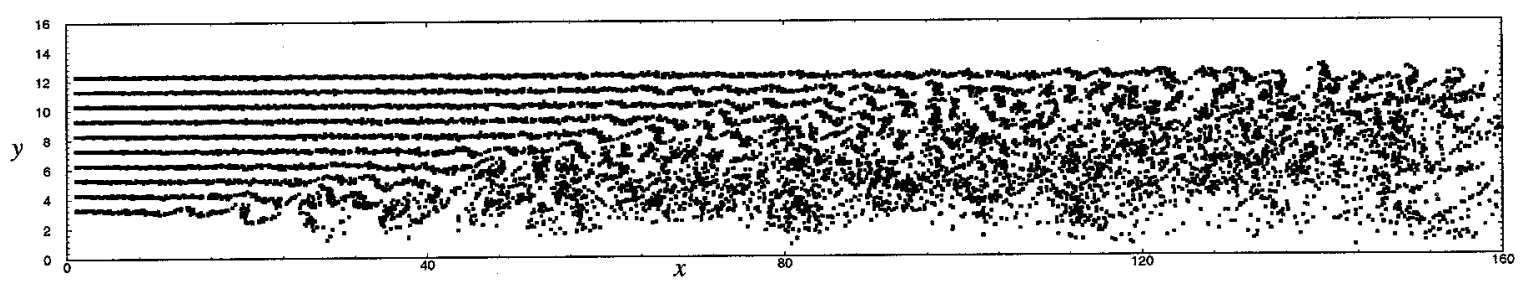

Figure 4: Streaklines of the discrete vortices for $U_{c}=425 \mathrm{~cm} / \mathrm{s}$ and $d U / d y=75 \mathrm{~s}^{-1}$. 


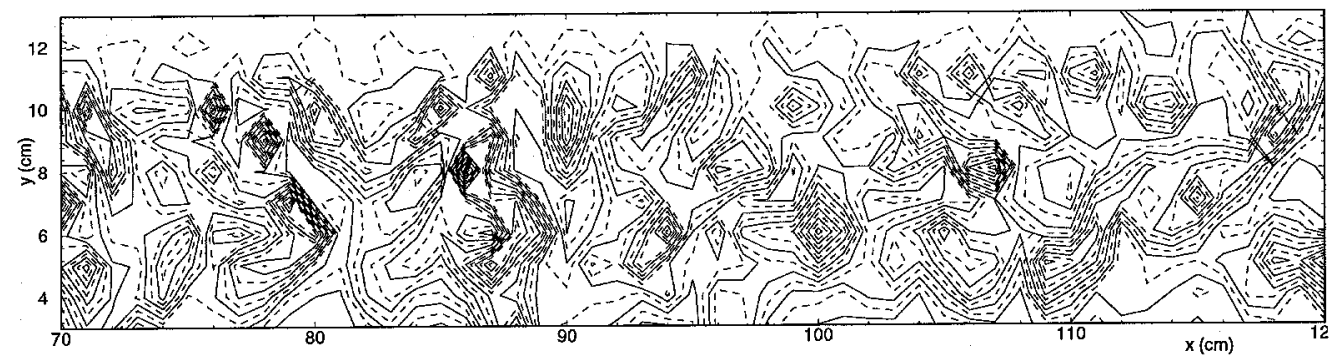

Figure 5: Vorticity contours for the flow field shown in Fig. 4.

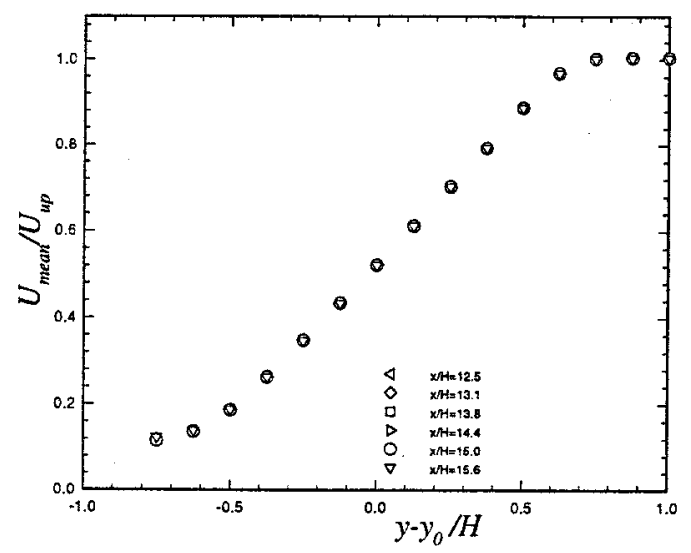

Figure 6: Normalized mean velocity profile at several downstream locations.

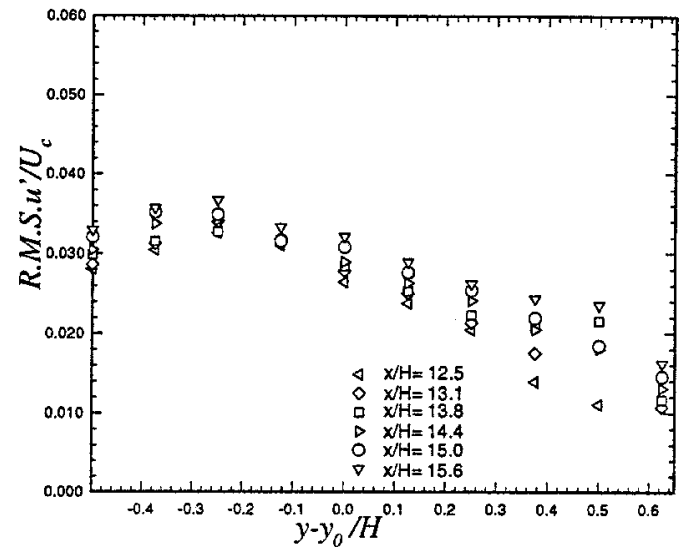

Figure 7: Normalized rms longitudinal velocity fluctuations versus normalized lateral direction. 


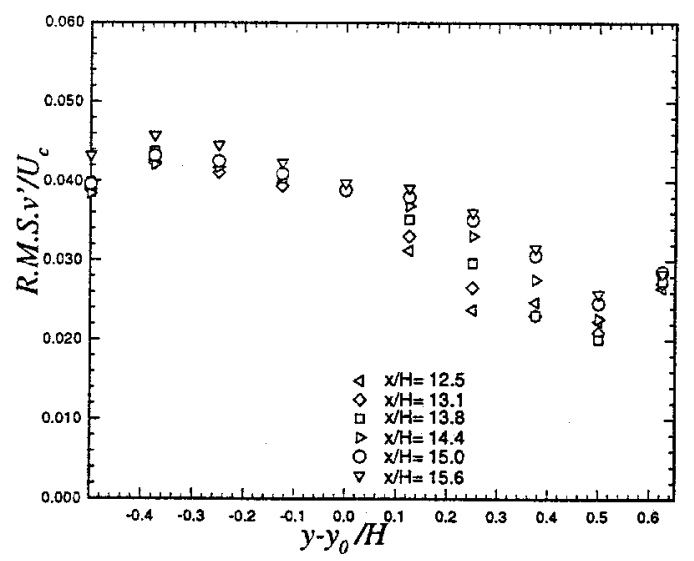

Figure 8: Normalized rms lateral velocity fluctuations vs. normalized lateral direction.

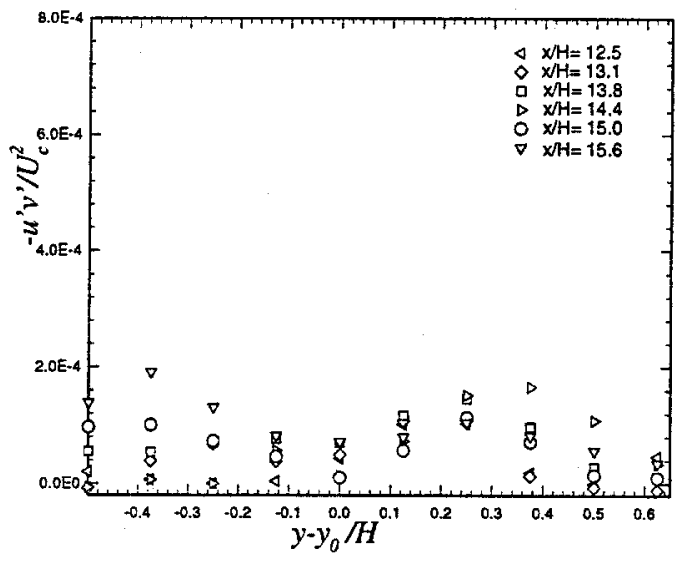

Figure 9: Normalized Reynolds shear stress vs. normalized lateral direction.

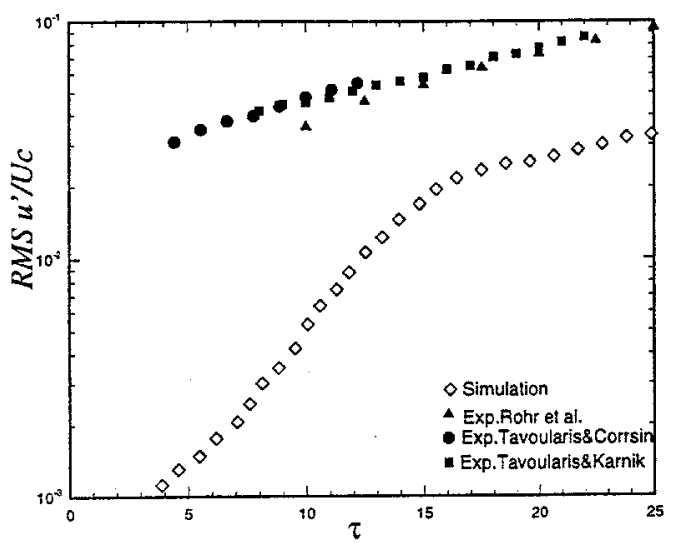

Figure 10: Downstream growth of the rms $u^{\prime}$ at the centerline vs. non-dimensional time. 


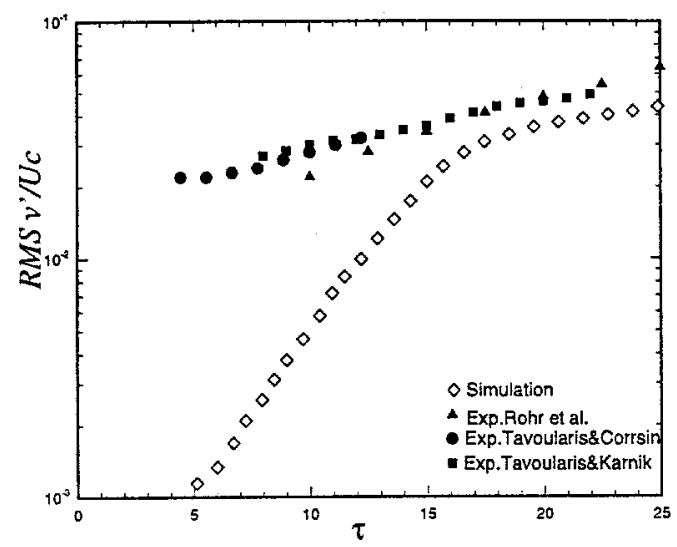

Figure 11: Downstream growth of the rms $v^{\prime}$ at the centerline vs. non-dimensional time.

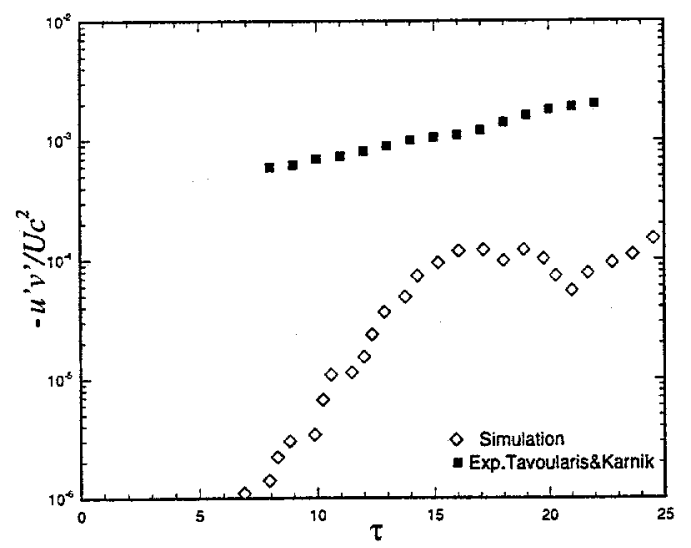

Figure 12: Downstream growth of the Reynolds shear stress at the centerline vs. non-dimensional time.

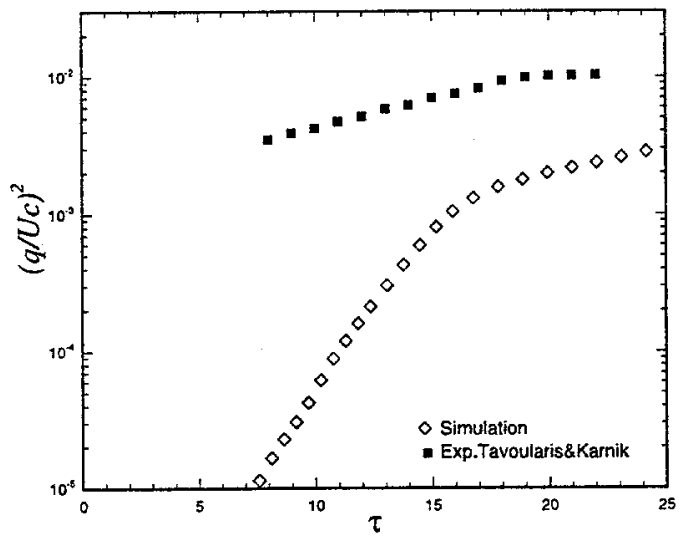

Figure 13: Downstream growth of turbulent kinetic energy at the centerline vs. non-dimensional time. 


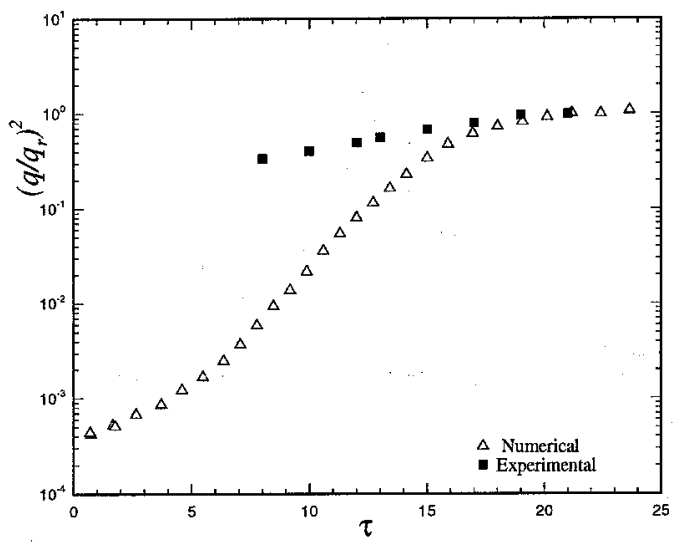

Figure 14: Non-dimensional turbulent kinetic energy at centerline versus $\tau$.

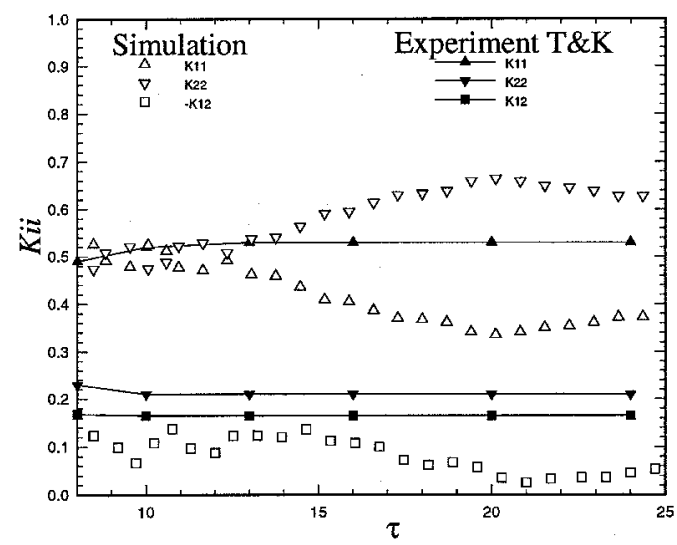

Figure 15: Downstream development of the fractional energy components. 